\title{
A Broadband Model of a Potential Transformer
}

\author{
Guishu Liang ${ }^{1}$, Xixiao Liu ${ }^{*}, 1$, Shuwei Zhang ${ }^{2}$ and Feng Gao ${ }^{3}$ \\ ${ }^{1}$ Hebei Provincial Key Laboratory of Power Transmission Equipment Security Defense, North China Electric Power \\ University, Baoding, 071000, China \\ ${ }^{2}$ State Grid Jixi Electric Power Company, Jixi, 158100, China \\ ${ }^{3}$ State Grid Shijiazhuang Electric Power Company, Shijiazhuang, 050000, China
}

\begin{abstract}
In this paper, a broadband circuit model of a potential transformer (PT) based on fractional calculus and circuit augmentation method was proposed to improve the high frequency characteristic while providing physical significance of the existing traditional equivalent circuit model with simple structure. In order to comprehensively reflect on the characteristics of hysteresis of the iron core, a fractional model of the magnetic hysteresis loop in iron core measured by a no-load experiment was set up instead of the single-value magnetization curve of previous model. Besides, the original equivalent circuit model was calculated at low frequencies, and the circuit augmentation method (CAM) was employed to extend the compatibility of the original equivalent circuit model in the wide range of frequency. The element values were calculated by the Brune method of synthesis. For validity, comparisons between the simulation and experiment of a $10 \mathrm{kV}$ single-phase oil-immersed PT with lightning overvoltage and switching overvoltage were conducted, respectively. The results showed that the broadband model presented in the paper had good accuracy while reserving the physical significance of original equivalent circuit model.
\end{abstract}

Keywords: Broadband modeling, Hysteresis, nonlinear, Network synthesis, Potential transformer.

\section{INTRODUCTION}

Power transformer is one of the most important and critical devices in power systems $[1,2]$. There are many kinds of transformer-like devices such as generator transformer, main transformer, potential transformers (PT) and current transformers (CT), etc. Potential transformer (PT) plays an important role in the field of power system measurement and protection. Traditional equivalent circuit model with simple structure and explicit physical significance correlate merely well with the measured parameters at lower frequencies. However, it deviates at higher frequencies (e.g. lightning overvoltage and switching overvoltage), therefore, the existing models cannot meet the stringent demand of production and movement. It is of great theoretical significance and practical value to set up an accurately broadband model.

There are several kinds of potential transformer models in use since decades [3-9]. In general, there are three ways of device modeling. The first one is pure physical method based on the specific structure of the devices, which requires the details about the structure and solve the complex electromagnetic field problems. The second one is the blackbox method according to the characteristics of external ports. The third one is the gray-box method. However, the existing

*Address correspondence to this author at the Hebei Provincial Key Laboratory of Power Transmission Equipment Security Defense, North China Electric Power University, Baoding, China; Tel: 8613483205813 ; E-mail: doolxx@163.com transformer modeling methods are only the first two, i.e. detailed model and black-box methods. On one hand, the detailed model of the transformer, valid for dozens of megahertz frequency range, requires that each turn should be represented by all mutual inductance coupling with each turn, and self-induction and self-capacitance. Solving such a model is very time-consuming. On the other hand, black-box model is a pure mathematical method which cannot reflect the physical significance of equipments. In this paper, the third one was used to establish the broadband circuit model for PT.

The iron core is a nonlinear element under the action of a large signal. There are several key factors such as magnetic saturation, hysteresis and eddy current for the nonlinear phenomena of iron core. However, it is difficult to set up a model having high accuracy for the analysis of Ferroresonant circuit. For modeling of the core excitation system, there have been some achievements since 1960. Widger proposed using a rational fraction approximation to represent the magnetization curves in 1969 [10]. Chua and Stromsmoe established the hysteresis model for electronic circuit studies in 1970 [11]. Bailey and Talukdar established the hysteresis model for power system studies in 1976 [12], and Saito et al. applied it to the simulation of single phase parallel inverter. Lucas proposed using a non-integer power series to represent the magnetization curves in 1988 [13]. Lucas et al. proposed using nonlinear inductance and nonlinear resistors in parallel connection to simulate the iron core magnetization process 1992 [14]. However, the magnetization curves of existing models are single-valued. In order to reflect the characteristics 
of the hysteresis of the iron core completely, there is a need to model the whole magnetic hysteresis loop. In this paper, a fractional order model for the magnetic hysteresis loop of iron core was established.

\section{CIRCUIT AUGMENTATION METHOD}

Circuit augmentation method is a gray-box method, and its basic augmentation concept is shown in Fig. (1) $[9,15$, 16].

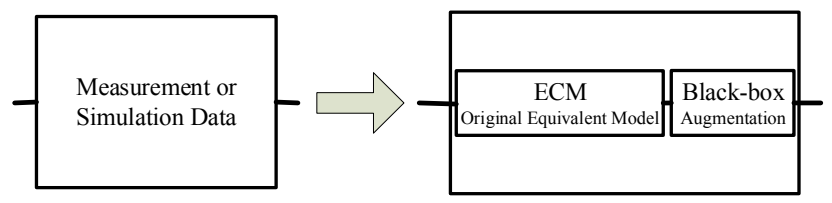

Fig. (1). Schematic of circuit augmentation method.

Among various kinds of augmentation methods, there are three common methods such as branch augmentation, parallel augmentation and cascade augmentation, as shown in Fig. (2).

(a)

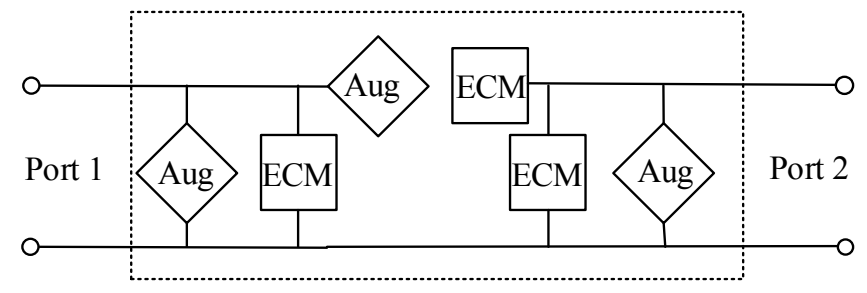

(b)

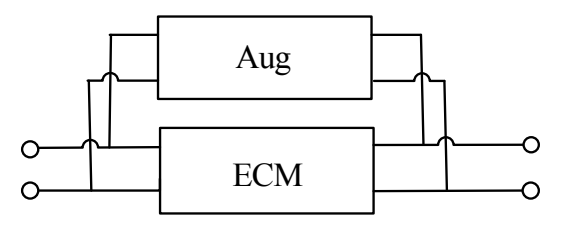

(c)

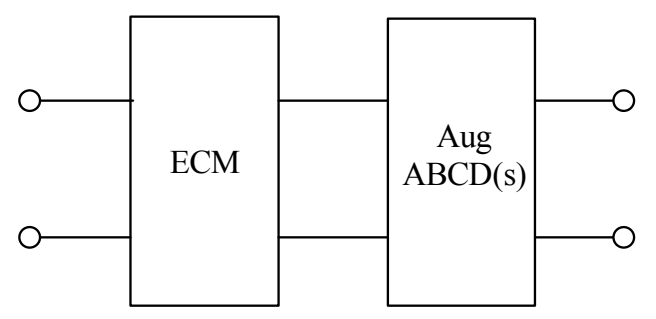

Fig. (2). Schematic of three common augmentation methods. (a) branch augmentation, (b) parallel augmentation, (c) cascade augmentation.

For m-port networks, the modified nodal analysis (MNA) equations $[9,14,15]$ are shown below:

$\mathbf{A}(j \omega) x=\mathbf{B} v$

where $\mathbf{A}(j \omega) \in \mathbb{C}^{N \times N}$ is the MNA matrix in the frequency $\omega$ , $\mathbf{B} \in \mathbb{R}^{N \times m}$ is a selection matrix which introduces the port voltages into the node space of MNA equations and $\mathbf{B}=[0,1]^{\mathrm{T}}, \mathbf{v} \in \mathbb{C}^{m \times 1}$ is a column vector composed by port voltages. $\mathbf{x}=\left[\begin{array}{l}\mathbf{x}_{1} \\ \mathbf{x}_{2}\end{array}\right] \in \mathbb{C}^{N \times 1}$

where $\mathbf{x}_{1} \in \mathbb{C}^{(N-m) \times 1}$ is a column vector composed of node voltages and additional currents, and $\mathbf{x}_{2} \in \mathbb{C}^{m \times 1}$ is a column vector composed of port currents.

From formula (1), the short-circuit admittance matrix of the $\mathrm{m}$-port equivalent circuit is obtained.

$\mathbf{Y}=\mathbf{B}^{\mathrm{T}} \mathbf{A}^{-1} \mathbf{B}$

$\mathbf{Y}_{\text {meas }}$ is used to denote the measured short-circuit admittance matrix. The purpose of circuit augmentation method is to obtain modified model $\hat{\mathbf{Y}}$ such that it matches the entire broadband spectrum by adding the corresponding branches $\Delta \mathbf{Y}$, i.e. $\hat{\mathbf{Y}}=\mathbf{Y}+\Delta \mathbf{Y}$.

Then, the following error function is obtained:

$$
\begin{aligned}
& \varepsilon(j \omega)=\left\|\mathbf{Y}_{\text {meas }}(j \omega)-\hat{\mathbf{Y}}(j \omega)\right\| \\
& \quad=\left\|\mathbf{Y}_{\text {meas }}(j \omega)-\mathbf{B}^{\mathrm{T}} \mathbf{A}^{-1} \mathbf{B}-\Delta \mathbf{Y}(j \omega)\right\|
\end{aligned}
$$

In this paper, the $\mathbf{Y}_{\text {meas }}$ parameters could also be indirectly deduced with scatting parameters $\mathbf{S}_{\text {meas }}$ measured by Agilent 4395A working as network analyzer, as shown in Fig. (3), and the measurement range of frequency with $87512 \mathrm{~A}$ was from DC to $2 \mathrm{GHz}$.

According to the two-port theory, the $\mathbf{Y}_{\text {meas }}$ parameter matrix of PT/CT can be obtained from the $\mathbf{S}_{\text {meas }}$.

$$
\begin{aligned}
& \mathrm{Y}=\left[\begin{array}{cc}
\mathrm{Y}_{11} & \mathrm{Y}_{12} \\
\mathrm{Y}_{21} & \mathrm{Y}_{22}
\end{array}\right]=[R(\mathbf{E}+\mathrm{S})]^{-1}(\mathbf{E}-\mathrm{S}) \\
& =\frac{1}{Z_{0}} \frac{1}{\left(1+\mathrm{S}_{11}\right)\left(1+\mathrm{S}_{22}\right)-\mathrm{S}_{12} \mathrm{~S}_{21}} \times \\
& {\left[\begin{array}{cc}
\left(1-\mathrm{S}_{11}\right)\left(1+\mathrm{S}_{22}\right)+\mathrm{S}_{12} \mathrm{~S}_{21} & -2 \mathrm{~S}_{12} \\
-2 \mathrm{~S}_{21} & \left(1+\mathrm{S}_{11}\right)\left(1-\mathrm{S}_{22}\right)+\mathrm{S}_{12} \mathrm{~S}_{21}
\end{array}\right]}
\end{aligned}
$$

\section{ORIGINAL EQUIVALENT MODEL OF PT}

A JDJ-10 of $10 \mathrm{kV}$ single-phase oil-immersed PT was calculated and measured.

The iron core of electromagnetic voltage transformer is a typical nonlinear element operating under overvoltage or lightning overvoltage. Firstly, a fractional order model of iron core was established in the large signals. Experimental principles are shown in Fig. (4), where the primary side of $\mathrm{PT}$ is disconnected.

According to the law of electromagnetic induction and transformation ratio of $\mathrm{PT}$, following relation is obtained:

$\psi=\int_{0}^{t} u d t+C, \dot{\psi}=\frac{\dot{U}}{j \omega}$ and $i_{1}=u_{2} / n_{u 2} / n$

And the relationship between current and flux linkage can be calculated by experimental results. 
(a)

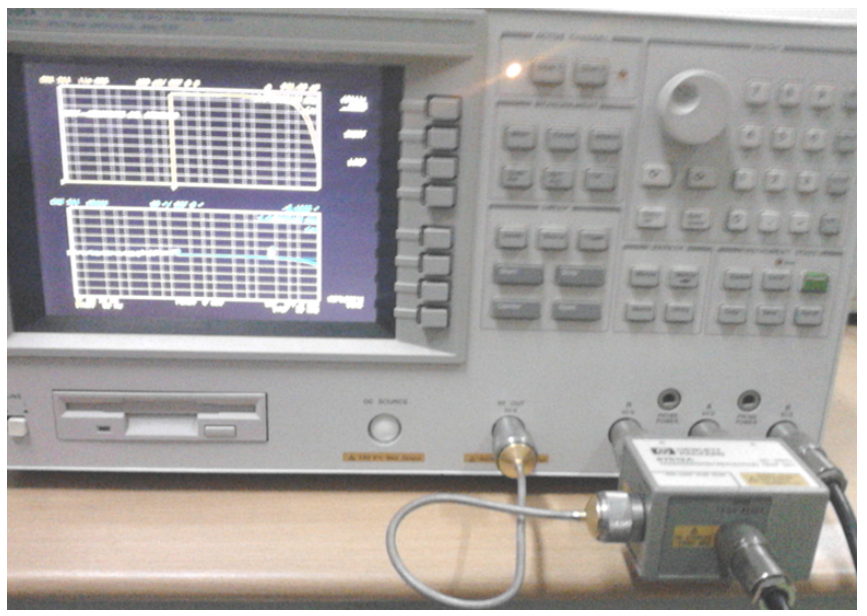

(b)

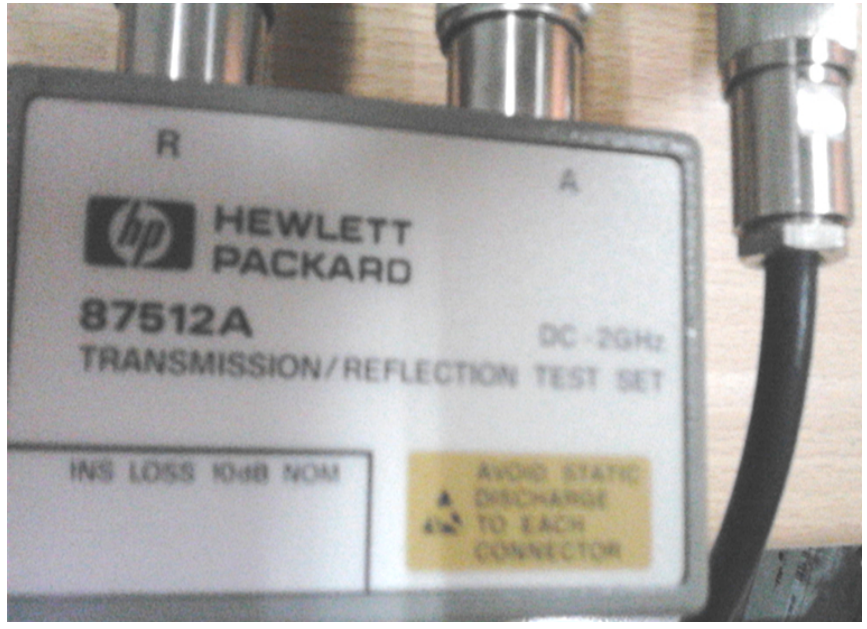

Fig. (3). Measuring equipments. (a) Agilent 4395A Analyzer, (b) $87512 \mathrm{~A}$

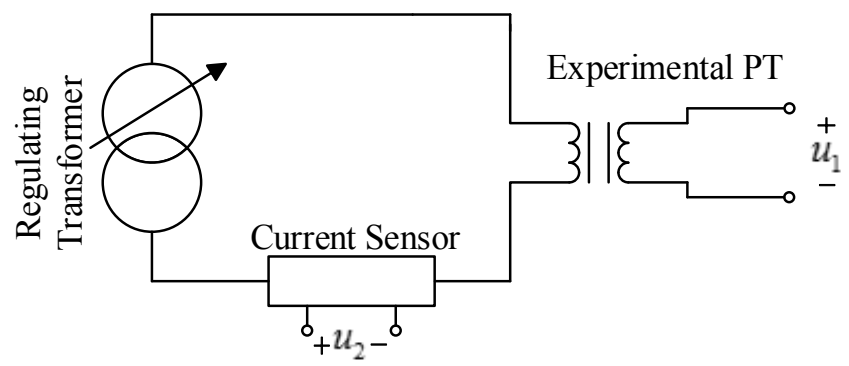

Fig. (4). No-load measurement of PT.

The basic principle describing the relationship between input and output in dynamic systems is as follows:

$$
\begin{aligned}
& y(t)+b_{1} \frac{d^{\beta_{1}}}{d t^{\beta_{1}}} y(t)+\cdots+b_{n} \frac{d^{\beta_{n}}}{d t^{\beta_{n}}} y(t) \\
& =a_{0} x(t)+a_{1} \frac{d^{\alpha_{1}}}{d t^{\alpha_{1}}} x(t)+\cdots+a_{m} \frac{d^{\alpha_{m}}}{d t^{\alpha_{m}}} x(t)
\end{aligned}
$$

After that the fractional polynomial of formula (8) was used to fit into the hysteresis loop

$$
B=a_{0} H+\sum_{k=1}^{N} a_{k} D^{\alpha_{k}} H
$$

Where $D^{\alpha} H=\frac{d^{\alpha} H}{d t^{\alpha}}, 0<\alpha<1$.

Combined with $B=\frac{k}{N S} \int u d t, H=\frac{N i}{L k}, u=\frac{d \lambda}{d t}, \quad$ the fitting result of flux linkage and current was obtained.

$u=a_{0} \frac{d i}{d t}+a_{1} \frac{d^{1+\alpha_{1}} i}{d t^{1+\alpha_{1}}}+a_{2} \frac{d^{1+\alpha_{2}} i}{d t^{1+\alpha_{2}}}+\cdots+a_{6} \frac{d^{1+\alpha_{6}} i}{d t^{1+\alpha_{6}}}$

The circuit is shown in Fig. (5), and for the sake of brevity, Fig. (6) was used . Results of comparison between measurement and simulation are shown in Fig. (7). In addition, the values of coefficient $a_{i}$ and their correspondent $\alpha_{i}$ are shown in Table 1.

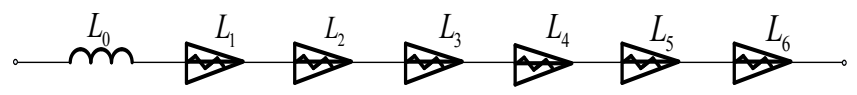

Fig. (5). Equivalent circuit of iron core.

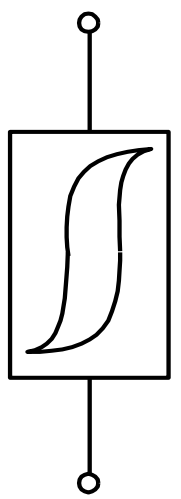

Fig. (6). Contracted notation.

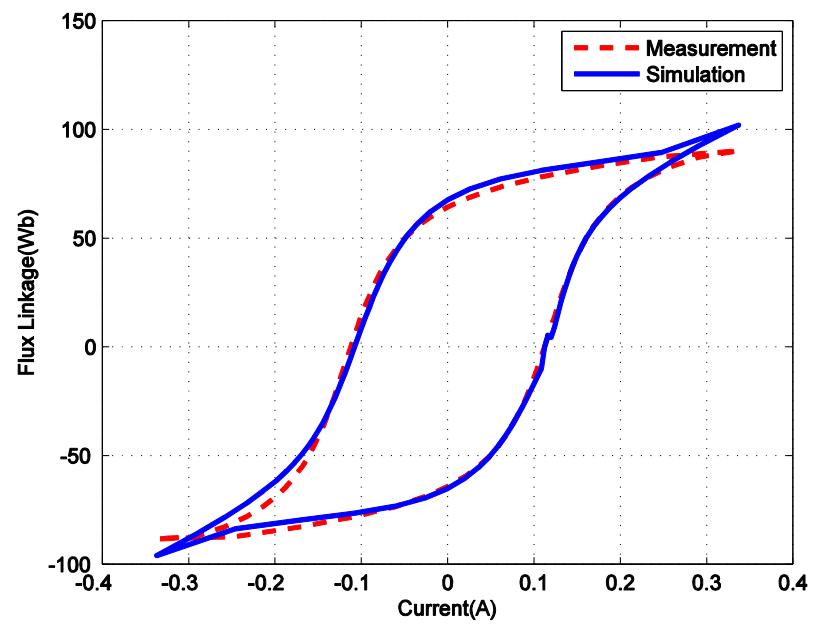

Fig. (7). Comparison result between measurement and simulation.

Using the Agilent 4395A Analyzer, the scattering parameters (S) were obtained, as shown in Fig. (8), and the transfer characteristic of PT is shown in Fig. (9).

At low frequency, the leakage inductance and resistance play a leading role. Because the signal of Agilent 4395A Analyzer was small-signal input, therefore the characteristic of the core was observed to be linear and the low frequency model of PT/two-winding transformer is shown in Fig. (10). 
Table 1. Coefficients and orders.

\begin{tabular}{|c|c|}
\hline Coefficient & Order \\
\hline \hline$a_{0}=-0.00245393050655755$ & null \\
\hline$a_{1}=-3.60492387516746$ & $\alpha_{1}=0.076804$ \\
\hline$a_{2}=-0.791579409744237$ & $\alpha_{2}=0.23351$ \\
\hline$a_{3}=1.87912230687602$ & $\alpha_{3}=0.47205$ \\
\hline$a_{4}=0.701165866198962$ & $\alpha_{4}=0.53542$ \\
\hline$a_{5}=-0.253480210360523$ & $\alpha_{5}=0.59612$ \\
\hline$a_{6}=0.0440853220991656$ & $\alpha_{6}=0.64206$ \\
\hline
\end{tabular}
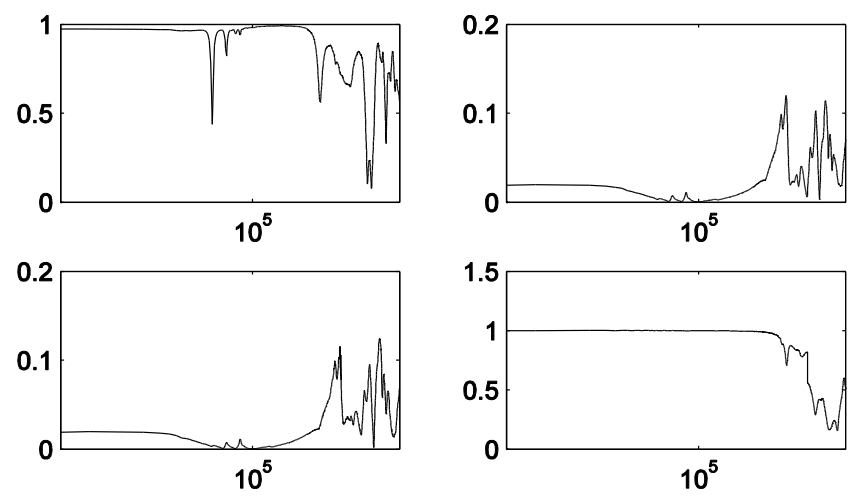

Fig. (8). Scattering parameters of PT.

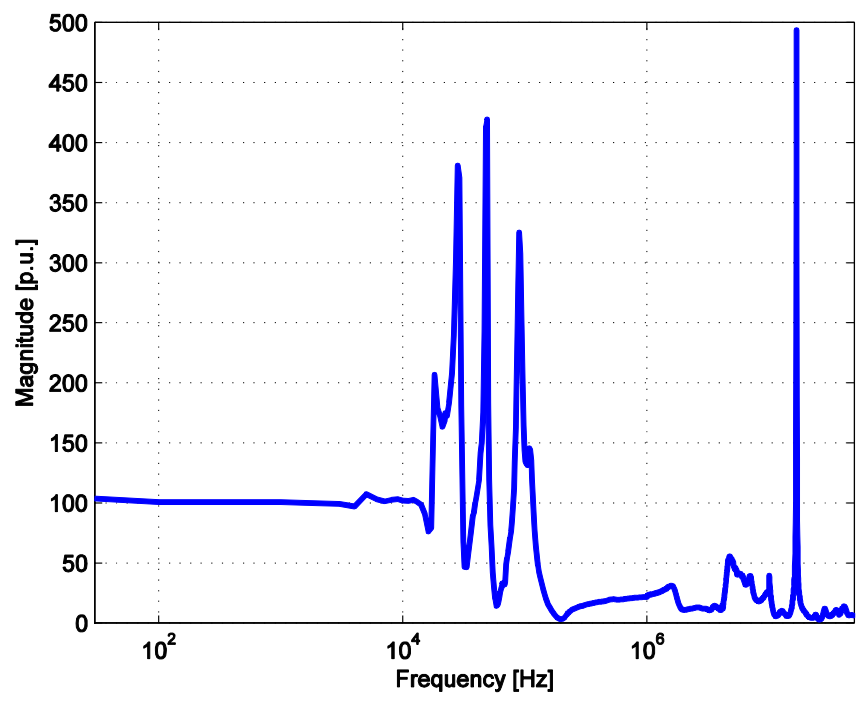

Fig. (9). Transfer characteristic of PT.

Therefore, the equivalent circuit in primary side can be calculated by

$Z_{11}-Z_{12}=R_{1}+j \omega L_{1}$ and $Z_{22}-Z_{12}=n^{2}\left(R_{2}+j \omega L_{2}\right)$

At medium frequency ( $\leqq 100 \mathrm{kHZ}$ ), the equivalent circuit model of PT/two-winding transformer can be shown in Fig. (11).

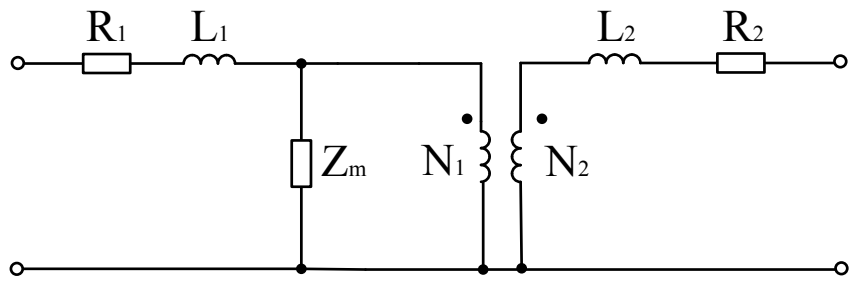

Fig. (10). Low frequency model of PT/two-winding transformer.

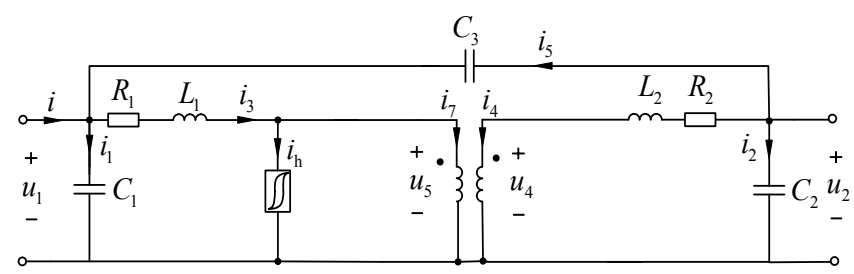

Fig. (11). Medium frequency model of PT/two-winding transformer.

$\mathbf{Y}$ is the admittance matrix, as shown in formula (11)

$\mathbf{Y}=\left[\begin{array}{cc}A_{22} B_{11}-A_{12} B_{21} & A_{22} B_{12}-A_{12} B_{22} \\ -A_{21} B_{11}+A_{11} B_{21} & -A_{21} B_{12}+A_{11} B_{22}\end{array}\right]$

Where $A_{11}=j \omega\left(L_{1}+Z_{m}+R_{1}\right), A_{12}=j \omega Z_{m}, A_{21}=j \omega Z_{m}$, $A_{22}=j \omega\left(n^{2} L_{2}+Z_{m}+n^{2} R_{2}\right)$,

$B_{11}=1-\omega^{2}\left[C_{3}\left(L_{1}+R_{1}\right)+C_{1}\left(L_{1}+R_{1}+Z_{m}\right)\right]$

$B_{12}=\omega^{2}\left[C_{3}\left(L_{1}+R_{1}\right)-Z_{m} \cdot C_{2}\right]$,

$B_{21}=\omega^{2}\left[C_{3}\left(n^{2} L_{2}+n^{2} R_{2}\right)-Z_{m} \cdot C_{1}\right]$

$B_{22}=1-\omega^{2}\left[C_{3}\left(n^{2} L_{2}+n^{2} R_{2}\right)+C_{2}\left(n^{2} L_{2}+n^{2} R_{2}+Z_{m}\right)\right]$.

Spurious capacitors can be calculated by formula (11).

Finally, the optimized element values were obtained with particle swarm optimization. The components of original equivalent circuit model were $C_{1}=0.15 \times 10^{-9} \mathrm{~F}$, $C_{2}=3.6607 \times 10^{-13} \mathrm{~F}, \quad C_{3}=0.89 \times 10^{-2} \mathrm{~F}, \quad R_{1}=1.6271 \Omega$, $R_{2}=0.00126 \Omega, L_{1}=3.5272 \mathrm{H}$, and $L_{2}=0.0089 \mathrm{H}, \mathrm{r}$.

\section{AUGMENTATION BRANCHES}

The spurious capacitors shown in Fig. (11) can be regarded as demonstrating a delta connection and meanwhile the rest shown in Fig. (11) can be regarded as a star connection scheme. Using the star-delta switching, the spurious capacitors were simplified into rest. The original equivalent circuit model and its augmentation considered in this paper are shown in Fig. (12).

$\mathrm{Y}_{\text {Aug1 } 1}, \mathrm{Y}_{\text {Aug } 2}$ and $\mathrm{Y}_{\text {Aug } 3}$ can be calculated by the shortcircuit admittance $\mathbf{Y}=\left[\begin{array}{cc}Y_{11} & Y_{12} \\ Y_{21} & Y_{22}\end{array}\right]$ of original equivalent circuit model, as shown in formula (12) 
$\left\{\begin{array}{l}Y_{\text {aug1 } 1}\left(\omega_{n}\right)=Y_{11 \text {.meas }}\left(\omega_{n}\right)+Y_{21 \text {.meas }}\left(\omega_{n}\right)-Y_{11 . \text {.ccm }}\left(\omega_{n}\right)-Y_{21 \text {.ccm }}\left(\omega_{n}\right) \\ Y_{\text {aug } 2}\left(\omega_{n}\right)=Y_{12 \text {.meas }}\left(\omega_{n}\right)-Y_{12 \text {.ecm }}\left(\omega_{n}\right) \\ Y_{\text {aug } 3}\left(\omega_{n}\right)=Y_{22 \text {.meas }}\left(\omega_{n}\right)+Y_{12 \text {.meas }}\left(\omega_{n}\right)-Y_{22 \text {.cem }}\left(\omega_{n}\right)-Y_{12 \text {.ecm }}\left(\omega_{n}\right)\end{array}\right.$

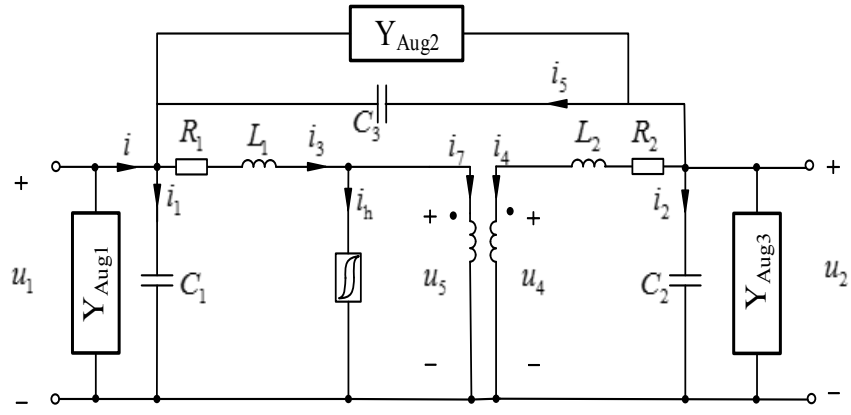

Fig. (12). The original equivalent circuit model and its augmentation .
The $Y_{\text {Aug }}$ can be expressed by the following rational fractional function

$Y_{\text {Aug }}(s)=\sum_{i=1}^{Q} \frac{k_{i}}{s-p_{i}}+d+s e$

Then the CAM model $Y_{\text {cam }}(s)$ with $\mathrm{Y}_{\text {Aug }}$ was obtained as shown below:

$$
\begin{aligned}
& Y_{\text {cam }}(s)=Y_{\text {ecm }}(s)+Y_{\text {Aug }}(s) \\
& \quad=Y_{\text {ecm }}(s)+\sum_{i=1}^{Q} \frac{k_{i}}{s-p_{i}}+d+s e
\end{aligned}
$$

Using the vector fitting technology [22], the formula (13) was obtained. Following this, the Brune method of synthesis $[17,18]$ was executed using the formula $(13)$, to make passivity correction before network synthesis if necessary. In this paper, the element values of Brune realizations are shown in Tables $\mathbf{2 - 4}$.

Table 2. Element values of brune realizations in $\boldsymbol{Y}_{A u g 1}$.

\begin{tabular}{|c|c|c|c|c|}
\hline Element Order Number & $\boldsymbol{R}_{\boldsymbol{i}}(\mathbf{O h m})$ & $\boldsymbol{L}_{\boldsymbol{p} i}(\mathbf{H})$ & $\boldsymbol{L}_{\boldsymbol{s i}}(\mathbf{H})$ & $\boldsymbol{C}_{\boldsymbol{i}}(\mathbf{F})$ \\
\hline \hline 1 & null & null & null & $3.63674300 \mathrm{e}-11$ \\
\hline 2 & 1.266848967 & $3.69518403 \mathrm{e}-5$ & $5.66089730 \mathrm{e}-5$ & $1.28305786 \mathrm{e}-11$ \\
\hline 3 & $1.878957500 \mathrm{e}+2$ & Null & null & $4.04340177 \mathrm{e}-11$ \\
\hline 4 & $4.396677378 \mathrm{e}+2$ & $4.57558350 \mathrm{e}-6$ & $5.17399620 \mathrm{e}-6$ & $1.03299110 \mathrm{e}-11$ \\
\hline 5 & $4.635046485 \mathrm{e}+1$ & $1.90412390 \mathrm{e}-5$ & $4.03330910 \mathrm{e}-4$ & $2.63177329 \mathrm{e}-11$ \\
\hline 6 & $2.169917945 \mathrm{e}+1$ & $2.67160370 \mathrm{e}-4$ & $2.66012790 \mathrm{e}-4$ & $3.48479594 \mathrm{e}-13$ \\
\hline 7 & $4.584115244 \mathrm{e}+1$ & $6.43917540 \mathrm{e}-4$ & $6.55973110 \mathrm{e}-4$ & $3.21056683 \mathrm{e}-13$ \\
\hline 8 & $4.181628600 \mathrm{e}+2$ & $2.86914197 \mathrm{e}-1$ & $1.67583400 \mathrm{e}-2$ & $4.02803017 \mathrm{e}-9$ \\
\hline 9 & $3.271638000 \mathrm{e}+2$ & $1.21779058 \mathrm{e}-5$ & $5.93108426 \mathrm{e}-6$ & $8.67269309 \mathrm{e}-12$ \\
\hline 10 & $9.050521138 \mathrm{e}+1$ & $1.30438753 \mathrm{e}-6$ & $8.64927476 \mathrm{e}-7$ & $3.544449687 \mathrm{e}-11$ \\
\hline 11 & $4.141604960 \mathrm{e}+1$ & null & null & $8.35087727 \mathrm{e}-10$ \\
\hline
\end{tabular}

Table 3. Element values of brune realizations in $\boldsymbol{Y}_{\text {Aug2 }}$.

\begin{tabular}{|c|c|c|c|c|}
\hline Element Order Number & $\boldsymbol{R}_{\boldsymbol{i}}(\mathbf{O h m})$ & $\boldsymbol{L}_{\boldsymbol{p} i}(\mathbf{H})$ & $\boldsymbol{L}_{\boldsymbol{s} i}(\mathbf{H})$ & $\boldsymbol{C}_{\boldsymbol{i}}(\mathbf{F})$ \\
\hline \hline 1 & null & null & $4.2215000100 \mathrm{e}-12$ \\
\hline 2 & $2.0595917846 \mathrm{e}+1$ & $3.3077103907 \mathrm{e}-5$ & $6.3022277009 \mathrm{e}-5$ & $1.7323021385 \mathrm{e}-12$ \\
\hline 3 & $4.4536451181 \mathrm{e}+1$ & $8.0784349644 \mathrm{e}-4$ & $2.0938785210 \mathrm{e}-3$ & $6.3180000000 \mathrm{e}-12$ \\
\hline 4 & $5.9765143964 \mathrm{e}+1$ & $1.4388813239 \mathrm{e}-4$ & $1.4312993211 \mathrm{e}-4$ & $3.7443352352 \mathrm{e}-13$ \\
\hline 5 & $9.8766379800 \mathrm{e}+2$ & $2.3113554230 \mathrm{e}-3$ & $2.6686902690 \mathrm{e}-3$ & $7.4471784533 \mathrm{e}-14$ \\
\hline 6 & $4.1011033900 \mathrm{e}+2$ & $1.7646411941 \mathrm{e}-4$ & $1.3081257294 \mathrm{e}-4$ & $3.2111664597 \mathrm{e}-12$ \\
\hline 7 & $9.3037759100 \mathrm{e}+2$ & $1.1106565872 \mathrm{e}-4$ & $8.8615102760 \mathrm{e}-5$ & $9.6379891693 \mathrm{e}-13$ \\
\hline 8 & $4.4236771300 \mathrm{e}+2$ & $3.6690638190 \mathrm{e}-5$ & $1.0851617050 \mathrm{e}-3$ & $3.8613043587 \mathrm{e}-12$ \\
\hline 9 & $1.0939862600 \mathrm{e}+3$ & 1.2038336188 & 1.4828517968 & $6.5019597701 \mathrm{e}-12$ \\
\hline 10 & $6.6173770900 \mathrm{e}+2$ & 4.4739023715 & 6.1202025241 & $2.0319866132 \mathrm{e}-11$ \\
\hline 11 & $1.5112170100 \mathrm{e}+4$ & 1.1934188199 & 1.3377369747 & $1.8605827939 \mathrm{e}-11$ \\
\hline 12 & $2.9523395100 \mathrm{e}+5$ & null & null & Null \\
\hline
\end{tabular}


Table 4. Element Values of Brune Realizations in $\boldsymbol{Y}_{\text {Aug3 }}$.

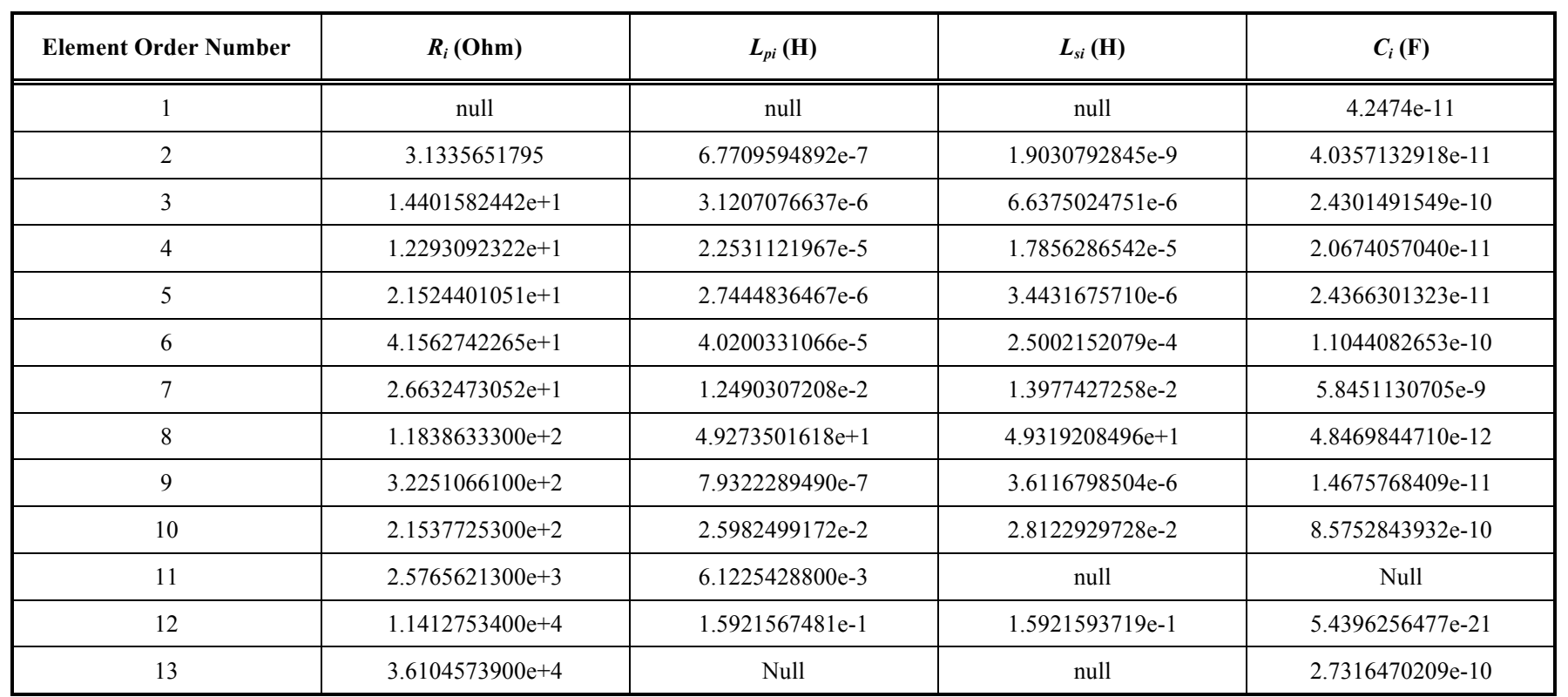

Furthermore, the global perturbed optimization [19-21] was achieved using PSpice, and the principle is shown in formula (15)

$Y_{\text {cam }}(s)=\underbrace{\sum_{i=1}^{Q_{c c m p}} \frac{\tilde{k}_{i}+\nabla \tilde{k}_{i}}{s-\left(\tilde{p}_{i}+\Delta \tilde{p}_{i}\right)}}_{\text {Disturbance Original Part }}+(\tilde{d}+\Delta \tilde{d})+\underbrace{\sum_{i=1}^{Q_{\text {aug }}} \frac{\hat{k}_{i}}{s-\hat{p}_{i}}+\hat{d}}_{\text {Augmentation Part }}$

\section{EXPERIMENTAL RESULTS}

The PT with lightning overvoltage and switching overvoltage were measured followed by comparison of the simulation result. The lightning overvoltage waveform is shown in Fig. (13), and the switching overvoltage is shown in Fig. (14).

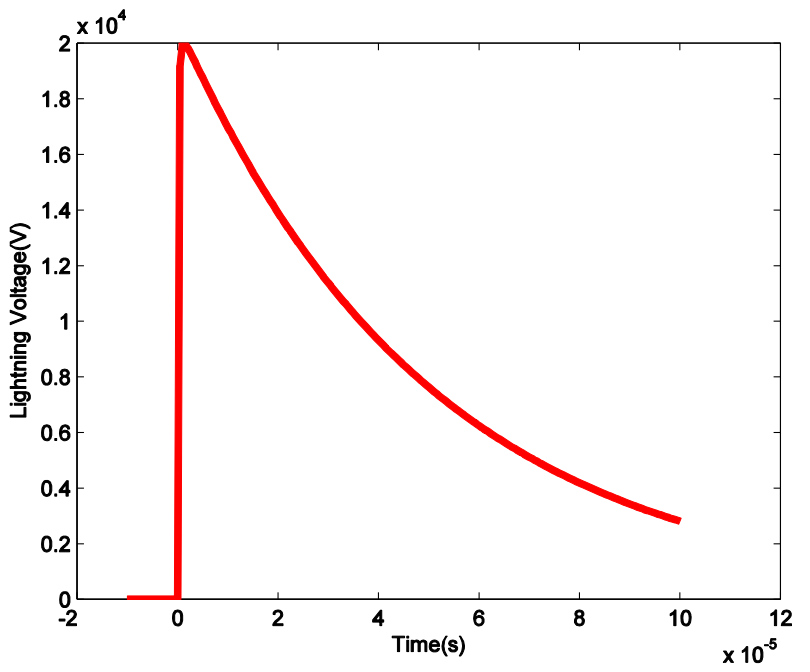

Fig. (13). Lightning overvoltage waveform.

As shown in Figs. $(15,16)$, through the comparison of measurement and simulation, the accuracy was observed to be satisfactory.

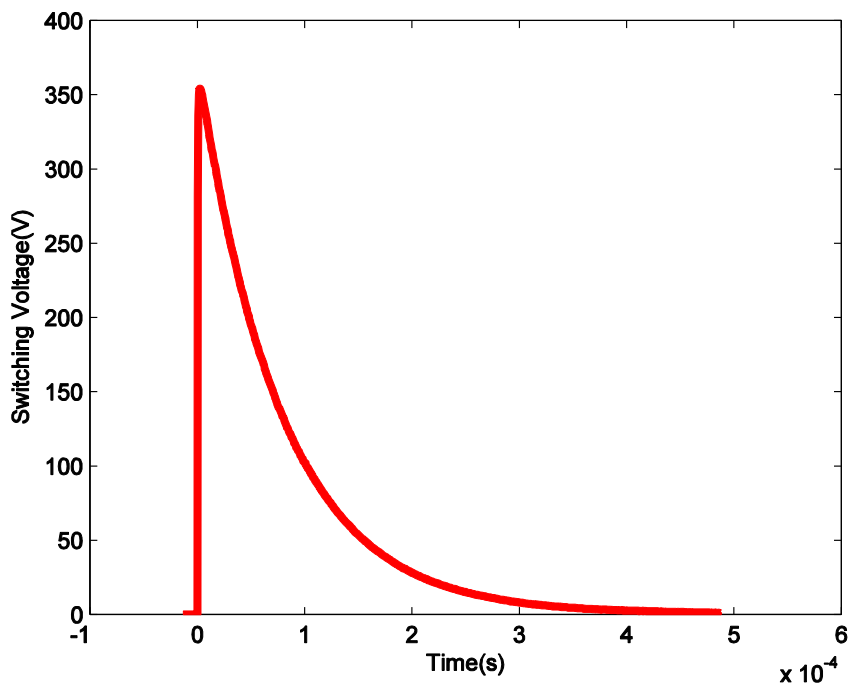

Fig. (14). Switching overvoltage waveform.

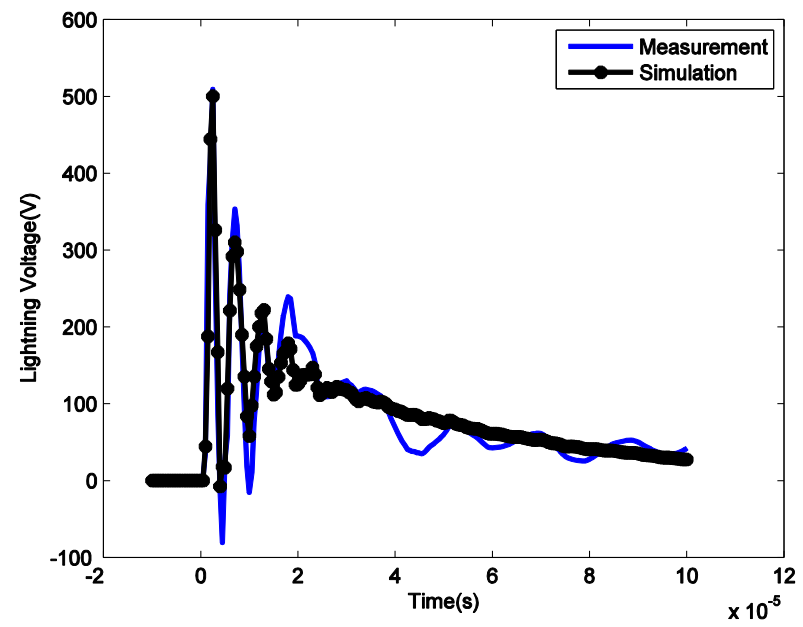

Fig. (15). Comparison under lightning overvoltage. 


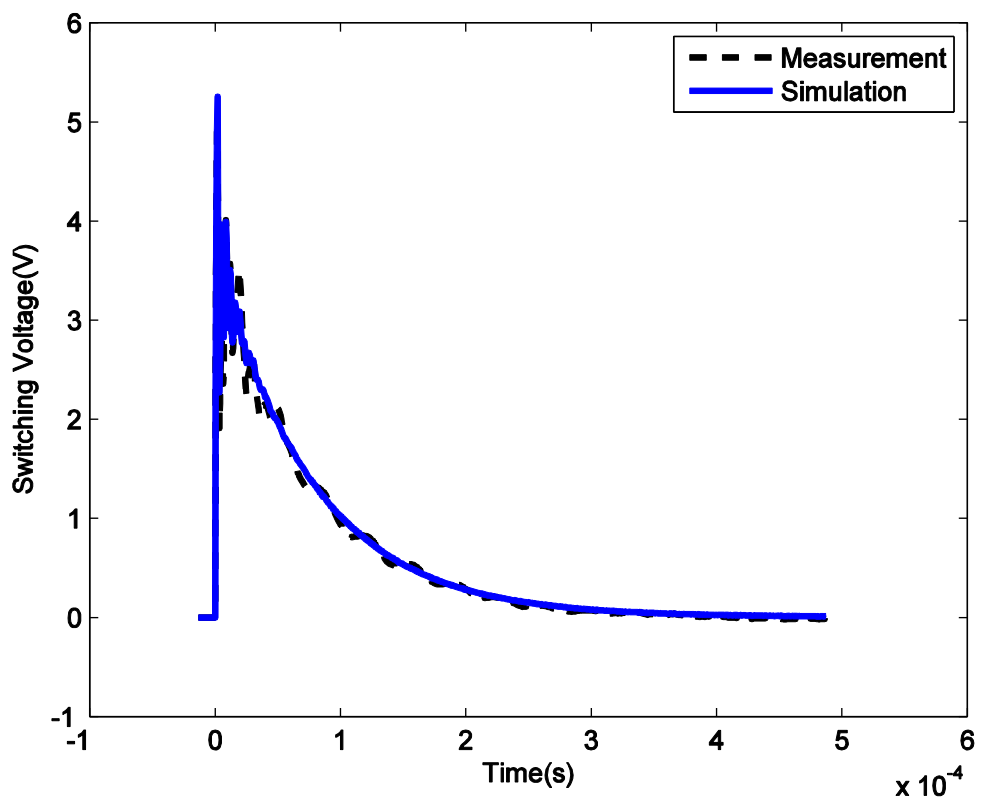

Fig. (16). Comparison under switching overvoltage.

\section{CONCLUSION}

In this paper, a broadband circuit model of PT was proposed for the power system measurement and protection in a wide range of frequencies. According to the no-load experiment data, the hysteresis loop curve was obtained to establish a fractional order model for the iron core of PT. Moreover, according to the $\mathrm{S}$ parameters measured by the Agilent 4395A analyzer with 87512A, the element's values were calculated using circuit augmentation method and Brune realization of synthesis for the augmented parts of PT.

To verify the performance of the proposed broadband model of the PT, experiments of lightning overvoltage and switching overvoltage were conducted. A good agreement was obtained between simulation and measurement under the lightning overvoltage and switching overvoltage respectively, and the results show that the proposed method enabled the engineers to retain the original equivalent circuit models while providing physical significance to obtain the high frequency characteristic accurately.

\section{CONFLICT OF INTEREST}

The authors confirm that this article content has no conflict of interest.

\section{ACKNOWLEDGEMENTS}

This research was supported in part by National Natural Science Foundation of China under Grant No. 51177048 and No.51207054, the Fundamental Research Funds for the Hebei Province Universities under Grant No. Z2011220, the Fundamental Research Funds for the Central Universities under Grant No. 11MG36 and No. 13MS75, and the Natural Science Foundation of Hebei Province under Grant No. E2012502009, respectively.

\section{REFERENCES}

[1] L. Van Der Sluis. Transients in Power Systems, Wiley, USA, 2001.

[2] J.H. Harlow, Ed., Electric Power Transformer Engineering, CRC press, USA, 2012

[3] Y. Liu, S. A. Sebo, and S. E. Wright, "Modeling of converter transformers using frequency domain terminal impedance measurement," IEEE Trans. Power Deliv., vol. 8, no. 1, pp. 66-72, 1993.

[4] A. Morched and L. Marti, "A high frequency transformer model for the EMTP," IEEE Trans. Power Deliv., vol. 8, no. 3, pp. 1655 $1625,1993$.

[5] J. R. Marti, J. R. Linares, and H. W. Dommel, "Current transformers and coupling-capacitor voltage transformers in realtime simulations," IEEE Trans. Power Deliv., vol. 12, no. 1, pp. 164-168, 1997.

[6] B. Gustavsen, "Wide band modeling of power transformers," IEEE Trans. Power Deliv., vol. 19, no. 1, pp. 414-422, 2004.

[7] Z. Zhang, F. Lv, and G. Liang, "A high-frequency circuit model of a potential transformer for the very fast transient simulation in GIS," IEEE Trans. Power Deliv., vol. 23, no. 4, pp. 1995-1999, 2008.

[8] J. Kolstad, C. Blevins, J.M. Dunn, and A. Weishaar, "A new circuit augmentation method for modeling of interconnects and passive components.", IEEE Trans. Adv. Packaging, vol. 29, no. 1, pp. 6777, 2006

[9] D. Paul, M.S. Nakhla, R. Achar, and A. Weisshaar, "Broadband modeling of high-Frequency microwave devices." IEEE Trans. Microwav. Theory Tech., vol. 57, no. 2, pp. 361-373, 2009.

[10] C.F.T. Widger, "Representation of magnetisation curves over extensive range by rational-fraction approximations." In: Proceedings of the Institution of Electrical Engineers, vol. 116. no. 1. IEE, 1969.

[11] L.O. Chua, and K.A. Stromsmoe, "Lumped-circuit models for nonlinear inductors exhibiting hysteresis loops." IEEE Trans. Circuit Theory, vol. 17, no. 4, pp. 564-574, 1970.

[12] S.N. Talukdar and J.R. Bailey, "Hysteresis Model for System Studies”, IEEE Trans. Power Apparatus Syst., vol. 95, no. 4, pp. 564-574, 1976.

[13] J.R. Lucas. "Representation of magnetisation curves over a wide region using a non-integer power series," Int. J. Elect. Eng. Educ., vol. 25 , no. 4 , pp. $335-340,1988$.

[14] J. R. Lucas, P.G. Maclaren, W.W.L. Keerthipala, and R.P. Jayasinghe, "Improved simulation models for current and voltage 
transformers in relay studies," IEEE Trans. Power Deliv., vol. 7, no. 1, pp. 152-159, 1992.

[15] J. Kolstad, C. Blevins, J. Dunn, and A. Weisshaar, "A new modeling methodology for passive components based on black-box augmentation combined with equivalent circuit perturbation.” In: Electrical Performance of Electronic Packaging, IEEE $13^{\text {th }}$ Topical Meeting on. IEEE, 2004.

[16] D. Paul, M.S. Nakhla, R. Achar, and A. Weisshaar, "An automated algorithm for broadband modeling of high-frequency microwave devices," In: Microwave Symposium Digest, IEEE MTT-S International. IEEE, 2006.

[17] E. A. Guillemin, Synthesis of Passive Networks, Wiley, New York, 1957.
[18] L. Weinberg, Network Analysis and Synthesis, McGraw-Hill, New York, 1962.

[19] R. Storn, and K. Price, "Differential evolution-a simple and efficient heuristic for global optimization over continuous spaces," J. Global Optim., vol. 11, no. 4, pp. 341-359, 1997.

[20] W. Huyer and A. Neumaier, "Global optimization by multilevel coordinate search." Journal of Global Optimization, vol. 14, no.4, pp. 331-355, 1999.

[21] X. Zhao, "A perturbed particle swarm algorithm for numerical optimization," Applied Soft Computing, vol.10, no. 1, pp. 119-124, 2010.

[22] B. Gustavsen, and A. Semlyen, "Rational approximation of frequency domain responses by vector fitting", IEEE Trans. Power Delivery, vol. 14, no. 3, pp. 1052-1061, July 1999.

(C) Liang et al.; Licensee Bentham Open.

This is an open access article licensed under the terms of the Creative Commons Attribution Non-Commercial License (http://creativecommons.org/licenses/by-nc/4.0/) which permits unrestricted, non-commercial use, distribution and reproduction in any medium, provided the work is properly cited. 